\title{
Combined CMR and catheterization data in determining right ventricular-arterial coupling in children and adolescents with pulmonary arterial hypertension
}

Uyen Truong $^{1 *}$, Sonali Patel ${ }^{1}$, Brian Fonseca ${ }^{1}$, Jamie Dunning ${ }^{2}$, Dunbar Ivy ${ }^{1}$, Robin Shandas ${ }^{1,2}$, Kendall Hunter $^{2}$

From 17th Annual SCMR Scientific Sessions

New Orleans, LA, USA. 16-19 January 2014

\section{Background}

Pulmonary arterial hypertension (PAH) remains a disease with high morbidity/mortality in pediatrics. Understanding ventricular-arterial coupling, a measure of how well matched the ventricular and vascular function is, may elucidate the pathway leading to right heart failure.

\section{Methods}

This retrospective study included subjects with PAH who a cardiac magnetic resonance (CMR) study within 14 days of cardiac catheterization between January 2009-August 2013. The effective elastance (Ea, index of arterial load) and right ventricular maximal end-systolic elastance (Emax, index of contractility) were determined by a combination of CMR and hemodynamic data. Ea is defined as (mean pulmonary arterial pressure minus pulmonary capillary wedge pressure)/stroke volume. Emax is defined as mean pulmonary arterial pressure/ end systolic volume. Ea/Emax ratio was derived. Additionally, a measure of non-invasive ventricular arterial coupling (assuming PWCP is insignificant, making Ea/ Emax = end systolic volume/stroke volume) was derived from only CMR. Pulmonary vascular resistance indexed (PVRi) and pulmonary vascular reactivity, as defined by Barst criteria (decrease in mean pulmonary artery pressure of $>20 \%$, unchanged/increased cardiac index, and decreased/unchanged pulmonary to systemic vascular resistance ratio), were also determined. Pearson correlation coefficients were calculated between PVRi and Ea, Emax, and Ea/Emax. Receiving operating characteristic

${ }^{1}$ Pediatric Cardiology, Children's Hospital Colorado, Aurora, Colorado, USA Full list of author information is available at the end of the article
(ROC) curve analysis determined the diagnostic value of $\mathrm{Ea} /$ Emax in predicting vascular reactivity.

\section{Results}

Sixteen subjects were identified for inclusion with equal gender distributions. Age ranged from 3 months to 23 years (mean $11.3+7.4$ years). Ea and Ea/Emax increased with increasing severity defined by PVRi, with $\mathrm{p}<0.001$ for both. Ea/Emax (range 0.43-2.82) was highly correlated with PVRi $(\mathrm{r}=0.92,95 \%$ CI 0.79-0.97, $\mathrm{p}<0.0001)$. Noninvasively derived ventricular arterial coupling was found

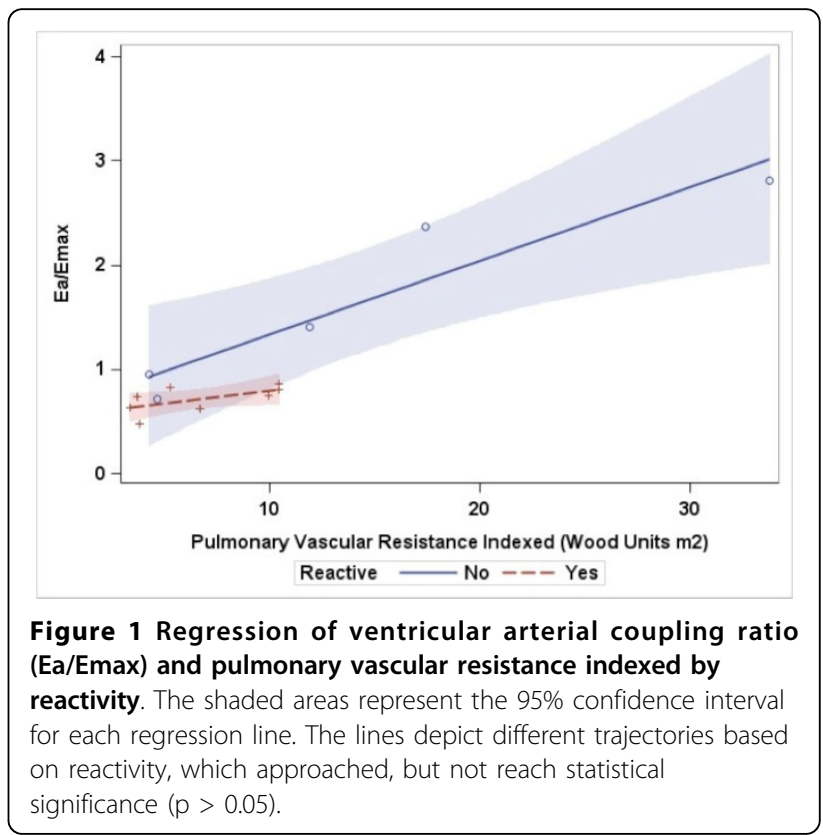




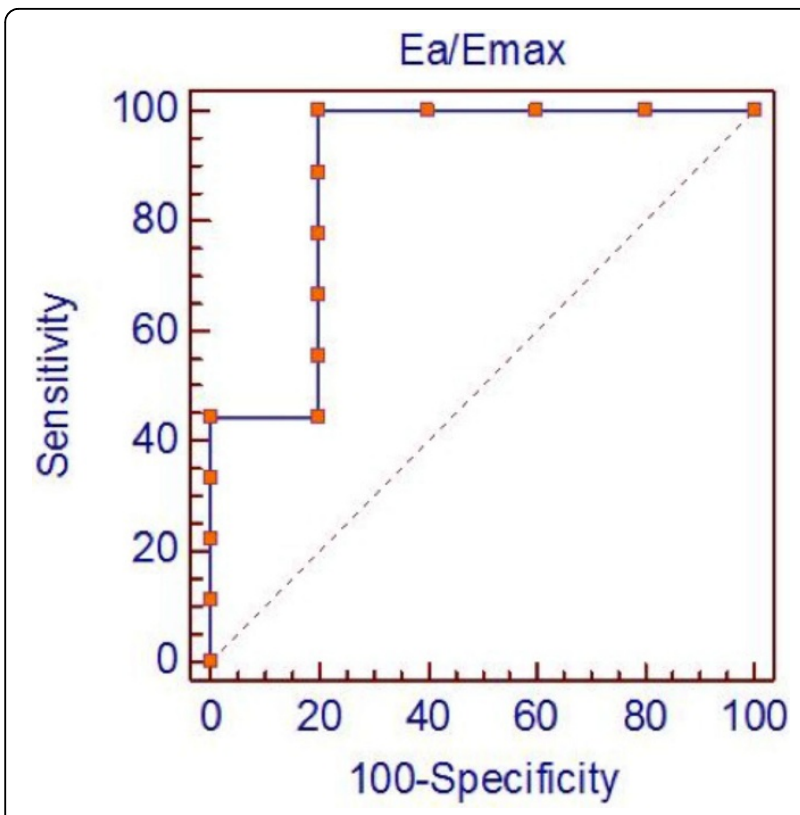

Figure 2 Receiver operating characteristic curve demonstrating an optimal threshold Ea/Emax ratio of 0.85 . Using this criterion, is associated with a sensitivity of $100 \%$ and a specificity of $80 \%$.

to be significantly correlated with PVRi $(\mathrm{r}=0.85,95 \% \mathrm{CI}$ 0.62-0.95, p $<0.0001$ ), but with a lower correlation coefficient than with $\mathrm{Ea} / \mathrm{Emax}$ derived from combined hemodynamic and CMR data. Regression of Ea/Emax and PVRi demonstrated differing lines when separated by reactivity, however, the lines were not significantly different (Figure 1). ROC curve analysis (Figure 2) revealed high accuracy of the Ea/Emax ratio in determining vascular reactivity. $\mathrm{Ea} /$ Emax of 0.85 had a sensitivity of $100 \%$ and a specificity of $80 \%$. The area under the curve is $0.89(\mathrm{p}=0.008)$, suggesting good discrimination between those who were and were not reactive.

\section{Conclusions}

Measurement of ventricular arterial coupling, Ea/Emax, in pediatrics is feasible. Pulmonary vascular non-reactivity may be due to ventricular-arterial decoupling in which ventricular contractility fails to parallel increasing afterload in severe PAH. Use of Ea/Emax may have significant prognostic implication.

\section{Funding}

This work was supported by UL1 TR000154 from NCATS/NIH, 5R01HL114753 from NHLBI/NIH, as well as K25-094749 and K24-081506.

\section{Authors' details}

${ }^{1}$ Pediatric Cardiology, Children's Hospital Colorado, Aurora, Colorado, USA ${ }^{2}$ Bioengineering, University of Colorado Denver Medical Campus, Aurora, Colorado, USA.
Published: 16 January 2014

doi:10.1186/1532-429X-16-S1-O43

Cite this article as: Truong et al: Combined CMR and catheterization data in determining right ventricular-arterial coupling in children and adolescents with pulmonary arterial hypertension. Journal of Cardiovascular Magnetic Resonance 2014 16(Suppl 1):043.
Submit your next manuscript to BioMed Central and take full advantage of:

- Convenient online submission

- Thorough peer review

- No space constraints or color figure charges

- Immediate publication on acceptance

- Inclusion in PubMed, CAS, Scopus and Google Scholar

- Research which is freely available for redistribution 